\title{
Convergence and rate of Convergence of of a system of exponential form difference equations
}

\author{
T Ibrahim ${ }^{1}$
}

${ }^{1}$ Mansoura University

April 27, 2020

\begin{abstract}
We study the persistence, boundedness and unboundedness, existence and uniqueness of positive equilibrium point, local and global asymptotic stability, and rate of convergence of a system of exponential form difference equations
\end{abstract}

\section{Hosted file}

paper.pdf available at https://authorea.com/users/315205/articles/445567-convergence-and-rateof-convergence-of-of-a-system-of-exponential-form-difference-equations 